Cite this: J. Anal. At. Spectrom., 2014, 29, 599

Received 2nd October 2013 Accepted 20th November 2013 DOI: 10.1039/c3ja50331c

www.rsc.org/jaas

\section{Changes in breath carbon isotope composition as a potential biomarker of inflammatory acute phase response in mechanically ventilated pediatric patients $\dagger$ t}

\author{
Juan P. Boriosi, ${ }^{a}$ Dennis G. Maki, ${ }^{\text {b }}$ Rhonda A. Yngsdal-Krenz, ${ }^{c}$ Ellen R. Wald, ${ }^{a}$ \\ Warren P. Porter, $\S^{d}$ Mark E. Cook $\S^{e}$ and Daniel E. Bütz $\S^{* e}$
}

Sepsis is a leading cause of mortality in intensive care units. Animal studies have shown exhaled breath carbon isotope delta values (BDVs, i.e., ${ }^{13} \mathrm{CO}_{2} /{ }^{12} \mathrm{CO}_{2}$ delta value) to be a marker for the inflammatory acute phase response (APR). The purpose of this study was to determine the baseline variability of BDVs in mechanically ventilated pediatric patients with and without systemic inflammatory response syndrome (SIRS) and to correlate the BDV with clinical course over time. The study was an observational pilot study in a pediatric intensive care unit at an urban, tertiary care children's hospital. Seventeen mechanically ventilated pediatric patients underwent measurement of exhaled BDVs every 8 hours for 72 hours. The BDV was not statistically different between SIRS, No-SIRS and SIRS with shock. The mean BDV was significantly lower in subjects with active sepsis or trauma/post-op status compared to subjects with NoInfection/Trauma/Surgery (No-ITS) or septic shock. Trend analysis over time revealed that the No-ITS and ITS in recovery groups had positive slopes. Subjects who developed infections during the study and subjects who underwent shock had a negative trend over time. These results indicate that the BDV does not correlate well with the SIRS status. However, when patients are classified based on their inflammatory APR the BDV correlates with the severity of systemic inflammation. When monitored over time, changes in the BDV may correlate with changes in physiology related to fractionation during the APR to infection, trauma or due to altered macronutrient oxidation during episodes of septic shock.

\section{Introduction}

The systemic inflammatory response to a wide variety of insults has been termed systemic inflammatory response syndrome (SIRS). SIRS is nonspecific and develops following a severe biological insult such as infection, trauma, surgery,

${ }^{a}$ Department of Pediatrics, University of Wisconsin-Madison, 600 Highland Avenue, Madison, WI, 53792, USA

${ }^{b}$ Department of Medicine, University of Wisconsin-Madison, 600 Highland Avenue, Madison, WI, 53792, USA

'Respiratory Therapy, University of Wisconsin-Madison, 600 Highland Avenue, Madison, WI, 53792, USA

${ }^{d}$ Department of Zoology, University of Wisconsin-Madison, 1117 W. Johnson Street, Madison, WI, 53706, USA

${ }^{e}$ Department of Animal Sciences, University of Wisconsin-Madison, 600 Highland Avenue, Madison, WI, 53706, USA. E-mail: debutz@wisc.edu

$\dagger$ This work was performed at the University of Wisconsin-Madison.

\$ Electronic supplementary information (ESI) available. See DOI: 10.1039/c3ja50331c

$\S$ Conicts of interest and sources of funding: Daniel E. Bütz, Mark E. Cook, and Warren P. Porter have an ownership interest in Isomark, LLC, which has licensed the technology reported in this publication. Juan P. Boriosi received funding from Isomark, LLC to conduct this study. For the remaining authors none were declared. pancreatitis, or burns. ${ }^{1}$ SIRS occurs in $82 \%$ of patients admitted to pediatric intensive care units (PICUs), including $23 \%$ with sepsis (SIRS in the presence of or as a result of suspected or proven infection). ${ }^{2}$ Sepsis is the most common cause of death in infants and children in the world. In the United States, there were more than 47000 cases of severe sepsis in children aged 19 years or less in 1999. Hospital mortality among US children with severe sepsis was $9 \%$ in 1999 . The estimated annual total costs were $\$ 2.3$ billion in children in the US alone. ${ }^{3}$

Early detection of bacterial sepsis followed by immediate intervention is critical for successful outcomes to patient care. ${ }^{4}$ Current clinical methods are often unreliable in detecting early bacterial sepsis; accordingly, there is great interest in identifying laboratory methods for early detection of sepsis. Although several biomarkers are potentially useful in the diagnosis of bacterial sepsis, all of them have limitations. All blood based biomarkers require blood to be drawn, a procedure that is somewhat invasive. White blood cell (WBC) count and absolute neutrophil count are inexpensive but lack adequate sensitivity and specificity to detect bacterial sepsis. ${ }^{5}$ Measurement of C-reactive protein (CRP) is readily available and relatively inexpensive compared to measurement of procalcitonin (PCT), but CRP measurements are not sensitive enough to detect bacterial 


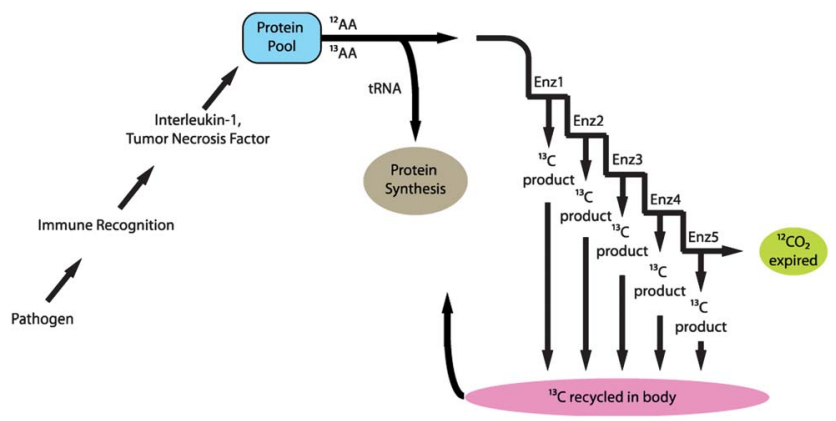

Fig. 1 Schematic representation of fractionation of carbon during the catabolic inflammatory acute phase response to infection.

sepsis early in its clinical course. ${ }^{6}$ PCT is a sensitive and early indicator of bacterial sepsis. However, measurement of PCT is expensive and requires a blood sample. ${ }^{7,8}$ Frequently PCT and CRP tests are used for predictors of sepsis outcome rather than as a means for early detection of infections.

Breath based markers, such as the carbon isotope breath delta value (BDV), may serve as better infection surveillance tools since they can be easily and non-invasively monitored. The BDV (i.e., ${ }^{13} \mathrm{CO}_{2} /{ }^{12} \mathrm{CO}_{2}$ delta value) has been shown to be a valuable biomarker of bacterial sepsis in different animal models. ${ }^{9}$ BDVs begin to drop immediately after administration of lipopolysaccharide (LPS) into experimental animals. The inflammatory APR induced by bacterial antigens initiates a complex cascade of events that result in the natural change in the ratio of ${ }^{13} \mathrm{CO}_{2} /{ }^{12} \mathrm{CO}_{2}$ (termed fractionation) due to the kinetic isotope effect (Fig. 1). This fractionation results in measureable changes in the BDV, which provides an avenue for early and non-invasive detection of sepsis through real-time monitoring. ${ }^{9-11}$ Furthermore, the change in BDV precedes other physiological changes associated with infection, such as drop in mean arterial pressure, hypoperfusion of the gut or alterations in blood oxygenation, carbon dioxide, and $\mathrm{pH} \cdot{ }^{12,13}$

There are no published studies evaluating the BDV as a marker of acute inflammation in humans. The purpose of this observational pilot study was to determine the baseline variability of exhaled BDVs in a population of mechanically ventilated pediatric patients and to correlate the trends in BDV over time with the severity of inflammation and clinical course.

\section{Experimental methods}

The Institutional Review Board at the University of WisconsinMadison approved the study. All patients in the PICU at American Family Children's Hospital were prospectively screened for participation in the present study. Informed consent was obtained for all participants. We enrolled mechanically ventilated (orotracheal, nasotracheal, and tracheotomy) patients, ages 1 month to 18 years, who were expected to remain ventilated for at least 72 hours. Seventeen subjects were enrolled and classified into groups (SIRS, No-SIRS and SIRS with shock) based on widely accepted criteria for pediatric SIRS ${ }^{14}$ and by severity of their underlying inflammatory APR at the time of enrollment: no infection, trauma or surgery (No-ITS; $n=6$ ); trauma or post-operative status ( $n=5$ ); active sepsis (SIRS in the presence of suspected or proven infection; $n=3$ ); and shock $(n=3)$. Shock was defined as subjects who have undergone hemodynamic dysfunction, such as vascular hypotension, with multiple organ failure. ${ }^{15}$ Subjects who have undergone a shock response were considered separately from active sepsis and/or SIRS due to a known alteration in the physiology of oxygen consumption at the cellular level ${ }^{15}$ that could impact macronutrient utilization and thus the BDV. ${ }^{13,16,17}$

All patients enrolled in this study underwent measurement of exhaled BDV at the time of study enrollment and every 8 hours thereafter for a total of 72 hours. Expiratory breath samples were collected from the ventilator exhaust by affixing a modified Rushe adaptor that created minimal positive pressure to fill the breath sample bag with expired air. Every effort was made to ensure that no changes from the standard of care in the ventilator function occurred. The breath samples obtained were considered as the average breath sample from the expiratory side of the ventilator circuit.

\section{Carbon isotope measurement}

Cavity ring-down spectroscopy (CRDS) was used to determine the BDV for all samples in the study. CRDS is a well-established technique for detecting trace quantities of gases. ${ }^{14}$ The key element in CRDS is a precisely aligned optical cavity with three very high reflectance mirrors (>99.995\%). Briefly, when light from a continuous wave laser that matches the resonance frequency of the cavity enters the cavity, the energy levels in the cavity increase over time. When the laser is turned off, the energy decays ("rings down") exponentially over microseconds; this decay is measured using a photodiode. CRDS measures the length of the ring down time over different wavelengths, because the laser is tuned for the analyte gas' molecular signature. A strongly absorbing gas will have a relatively short ring down time compared to an empty cavity. Each gas has its characteristic concentration-dependent ring down time at different wavelengths. Fig. 2 depicts a ring-down curve, showing the changing light intensity inside the cavity as a function of time after the laser is shut off. CRDS generates a detailed profile, area and height of an individual absorption feature

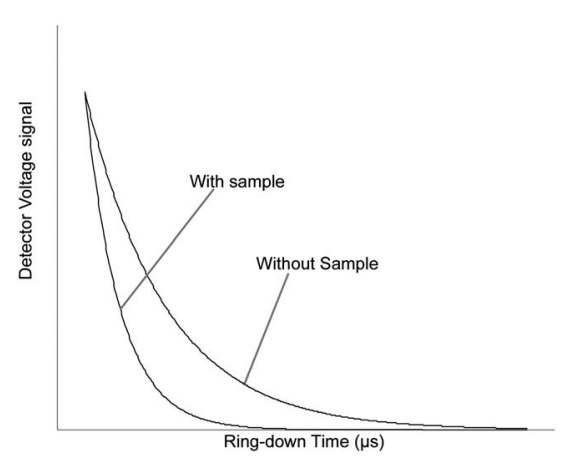

Fig. 2 Ring-down trace showing an exponential decay of the optical signal with and without analyte gas. 
(Fig. 3, panel A). The ${ }^{13} \mathrm{CO}_{2}$ delta value is determined by scanning the absorption frequency features for ${ }^{13} \mathrm{CO}_{2}$ and ${ }^{12} \mathrm{CO}_{2}$ separately and calculating the value referenced to Pee Dee Belemnite (PDB). Data are expressed as delta values or parts per million $(\%)$ :

$$
\delta=\frac{{ }^{13} \mathrm{CO}_{2} /{ }^{12} \mathrm{CO}_{2}(\text { sample })-{ }^{13} \mathrm{CO}_{2} /{ }^{12} \mathrm{CO}_{2}(\mathrm{PDB})}{{ }^{13} \mathrm{CO}_{2} /{ }^{12} \mathrm{CO}_{2}(\mathrm{PDB})} \times 1000
$$

The precision obtained for ${ }^{13} \mathrm{CO}_{2} /{ }^{12} \mathrm{CO}_{2}$ using the Picarro 2101i isotopic $\mathrm{CO}_{2}$ CRDS instrument used in this study is better than $0.3 \%$ with $\mathrm{CO}_{2}$ concentrations between 380 and $500 \mathrm{ppm}$ $\mathrm{CO}_{2}$ corresponding to a precision of $10 \mathrm{ppb}{ }^{13} \mathrm{CO}_{2}$ and $200 \mathrm{ppb}$ for ${ }^{12} \mathrm{CO}_{2}$ individually. Fig. 3, panel B, depicts isotopic precision (in box plots) of CRDS for determination of the isotope ratio of two $5 \% \mathrm{CO}_{2}$ reference gasses (Cambridge Isotope Labs, Tewksbury, MA) diluted to $400 \mathrm{ppm} \mathrm{CO}_{2}$ with zero air (i.e. air containing $>0.001 \mathrm{ppm} \mathrm{CO}_{2}$ ).

\section{Breath sample analysis}

Isomark, LLC analyzed all carbon samples. The expired breath was placed in a gas-tight bag with a de-identified sample number by a respiratory therapist at the point of care, and transported to Isomark for analysis. Breath sample bags were constructed of an edge sealed multi-layered plastic bag consisting of nylon/polyethylene-EVOH co-extrusion with an integrated high volume lure-valve for filling and sealing the bag. The bag was verified to maintain the carbon stable isotope ratio for greater than 72 hours. To ensure accurate measurements all breath samples were analyzed within the first 48 hours of collection, typically less than 24 hours from the time of collection. At the time of sample measurement some of the gas was withdrawn from the gas-tight bag and diluted to about $500 \mathrm{ppm}$ $\mathrm{CO}_{2}$ concentration using $\mathrm{CO}_{2}$ free air. The stable isotopes of carbon in $\mathrm{CO}_{2}$ were determined using an isotopic $\mathrm{CO}_{2}$ cavity ring-down spectrometer as described above.

A

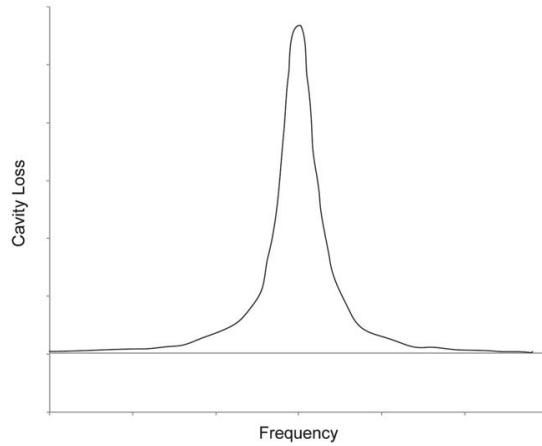

Fig. 3 Panel A: loss spectrum from WS-CRDS. The curve represents the cavity loss over a spectral line for a single analyte, for example ${ }^{13} \mathrm{CO}_{2}$. Panel $\mathrm{B}$ : box plot of the analyzer delta value precision for two reference samples.

\section{Statistics}

The BDV was analyzed in two ways, first by assessing the baseline variation in the BDV at the time of enrollment and second by assessing trends in the BDV over time. To assess the baseline variation subjects were classified into $\operatorname{SIRS}^{\mathbf{1 8}}(n=5)$, No-SIRS $(n=8)$ or SIRS with shock $(n=4)$ or by the severity of their underlying inflammatory APR at the time of enrollment based on the following categories: no infection, trauma or surgery (No-ITS); $(n=6)$; trauma or post-operative status ( $n=$ $5)$; active sepsis $(n=3)$; and shock septic $(n=3)$. The mean, range and interquartile range were calculated for each category. ANOVA with least significant difference post-hoc analysis was applied individually to both sets of diagnostic classification. To assess trends in the data over time the subjects were classified based on their clinical course with the following categories: stable with No-ITS, ITS with improving clinical condition, developing infection, and septic shock with organ failure. Best-fit linear trends were calculated using Microsoft Excel and only subjects who provided at least 9 consecutive samples were considered. The mean and standard deviation of the slope of the line were calculated and ANOVA with least significant difference post-hoc analysis was applied to the slope of the line. Additionally the trends in the BDV and daily SIRS criteria were assessed using the SAS statistical analysis package using the mixed model for repeated measures; ${ }^{19}$ least square means were used to compare each category with the stable No-ITS group. Differences were considered significant with a $p$-value $\leq 0.05$.

\section{Results}

We enrolled 17 mechanically ventilated pediatric patients ranging in age from 1.5 months to 17.5 years between July 2011 and January 2012 (Table 1; ESI Table 1\$). Nine out of 17 patients met SIRS criteria at the time of enrollment, 4 of which had undergone shock. The mean BDV was lower, though not significantly different, in the SIRS group compared to the nonSIRS and SIRS with shock groups at the time of enrollment (mean BDV in SIRS -22.7\% ; SE 1.1; No-SIRS -21.1\%; SE 0.8; SIRS with shock $-19.5 \%$; SE $0.9 ; p=0.14$; Fig. 4, panel A). However, the mean BDV was significantly lower in subjects with active sepsis or trauma/post-op status compared to subjects with No-ITS or septic shock (mean BDV of $-23.0 \%$ SE 1.3,

Table 1 Demographics

Patient characteristics

Gender

Age in years (range) Height in cm (range) Weight in $\mathrm{kg}$ (range) Ethnicity

\author{
9 male \\ 8 female \\ 7.3 years ( 1.5 months -17.5 years) \\ $107.4(42.8-175)$ \\ $39.4(3.4-116)$ \\ $70.6 \%$ white \\ $5.9 \%$ black \\ $5.9 \%$ native American \\ $17.6 \%$ decline to report
}




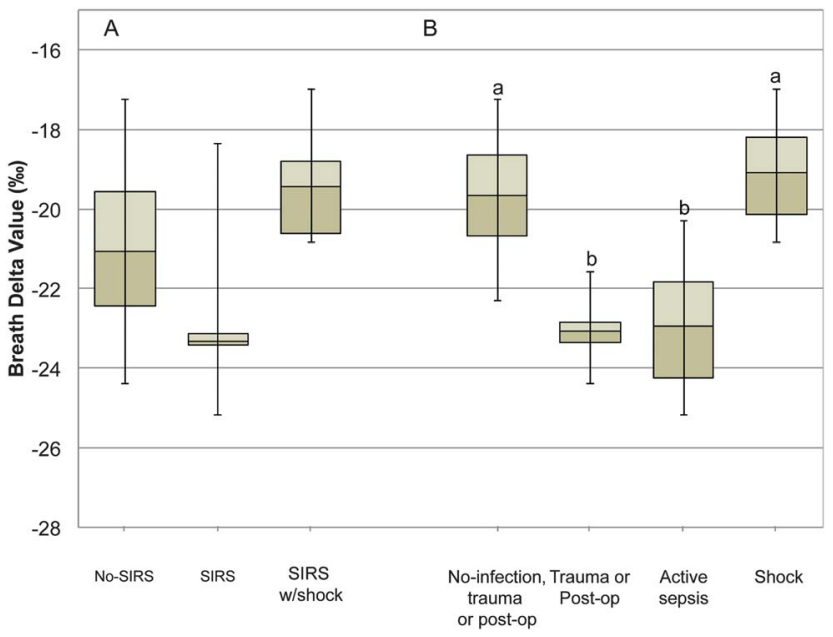

Fig. 4 The mean breath delta value (BDV) at the time of study enrollment of subjects classified as systemic inflammatory response syndrome (SIRS) or no systemic inflammatory response syndrome (No-SIRS) or SIRS with shock (panel A), and the mean BDV at the time of study enrollment of subjects classified based on the severity of their underlying inflammatory acute phase response (APR) (panel B). Data are presented as means with an interquartile range with error bars representing the range. ANOVA with least significant difference posthoc analysis was used to analyze the data, and letter superscripts $a$ and b represent significant differences with $p<0.05$.

$-23.1 \%$ SE $0.5,-19.7 \%$ o SE 0.7 , and $-19.1 \%$ SE 1.1 respectively; $p=0.01$; Fig. 4 , panel B). There was no statistical difference in the mean breath delta value between patients with No-ITS and patients with septic shock (Fig. 4, panel B).

Since it is hypothesized that the trend in the BDV over time may correlate with changes in physiology during the onset of infection, sepsis and septic shock we analyzed the trend in the BDV over the course of study participation. Only subjects who were able to provide at least 9 consecutive samples were included in the trend analysis. The BDV was plotted over time for each subject, and the slope of the linear trend line was assessed. The subjects were grouped as before in the No-ITS, ITS in a recovery trend, subjects who developed an infection during the study, and subjects who underwent septic shock (Fig. 5). The slope of the line was averaged for each group and analyzed by ANOVA. The mean slope of the line was positive for No-ITS subjects $(0.33,0.35 \mathrm{SD})$ and ITS subjects in the recovery phase. The mean slope of the line was negative for patients who developed infections during the study $(-0.56,0.13 \mathrm{SD})$, and for subjects who underwent septic shock $(-0.45,0.76 \mathrm{SD})$. When analyzed over time the No-ITS group had a stable BDV between -18 and $-19 \%$. The ITS subjects in recovery had a significantly more negative BDV at the start of the study that trended in a positive direction towards the No-ITS group and was no longer significantly different than the No-ITS group by the final sample. Subjects that underwent septic shock had a generally negative, but variable response in the BDV trend over time; however, these subjects were not significantly different from the No-ITS group. Subjects that developed infections during the study were initially not significantly different from the No-ITS group, but as subjects developed infections the BDV trended in

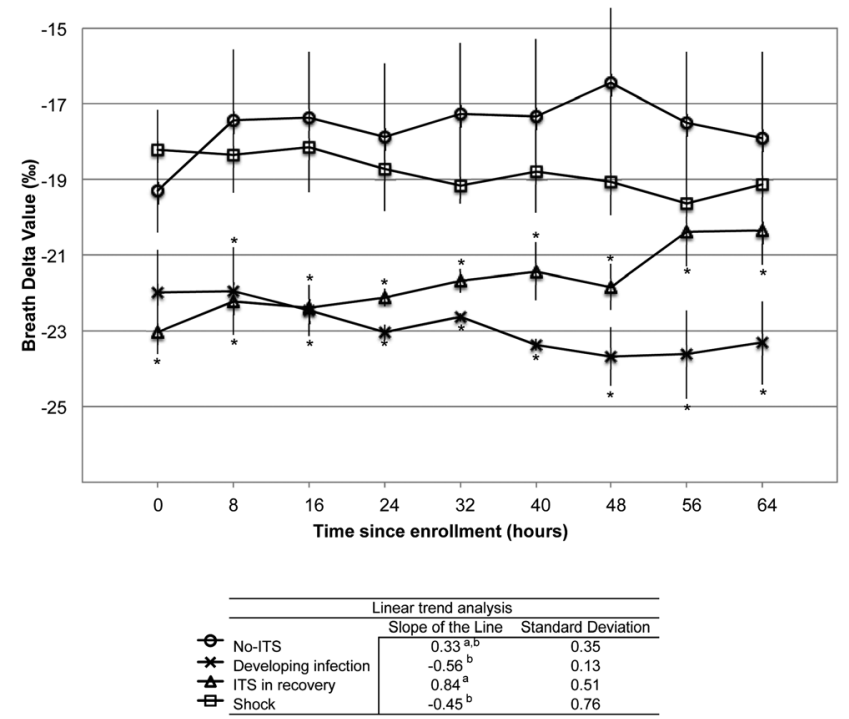

Fig. 5 Summary trend analysis of breath delta values (BDVs) over time. Subjects were classified based on their clinical course with the following categories: stable with No-ITS, ITS with improving clinical condition, developing infection, and septic shock with organ failure. Best-fit linear trends were calculated using Microsoft Excel and only subjects who provided at least 9 consecutive samples were considered. The mean and standard deviation of the slope of the line were calculated and ANOVA with least significant difference post-hoc analysis was applied to the slope of the line. Additionally the trends in the BDV and daily SIRS criteria were assessed using the SAS statistical analysis package using the mixed model for repeated measures; least square means were used to compare each category with the stable No-ITS group. Asterisks were used to signify significant differences from the No-ITS group in the repeated measures analysis, and letter superscripts were used to signify differences in the slope of the line. Differences were considered significant with a $p$-value $\leq 0.05$.

a negative direction and became significantly more negative than the No-ITS group.

We also evaluated the SIRS criteria over time for these subject groupings. Table 2 shows the mean and standard deviation of the SIRS criteria for each group of subjects. There were no statistical differences in the body temperature or heart rate. The respiratory rate was slightly depressed in the shock group on day 1 when compared to the No-ITS group. On day 2 the white blood cell (WBC) count was elevated in subjects who developed infections and in subjects who had undergone shock when compared to the No-ITS group. There were no other statistically significant differences on days 3 and 4 of the study. A detailed analysis of representative individual subjects' BDV, body temperature and WBC counts can be found in the ESI.

\section{Discussion}

Sepsis leading to shock is a major cause of mortality in adults and pediatric intensive care units. ${ }^{20}$ Early detection and prompt treatment of sepsis are critical for survival and optimal outcomes., ${ }^{\mathbf{4 2 1}}$ Based on preclinical animal studies $^{\mathbf{1 3 , 1 6}}$ we hypothesized that the breath delta value would be a sensitive marker of the inflammatory APR to infection in mechanically 
Table 2 Mean and standard deviation of systemic inflammatory response syndrome criteria for each group of subjects ${ }^{a}$

SIRS criteria

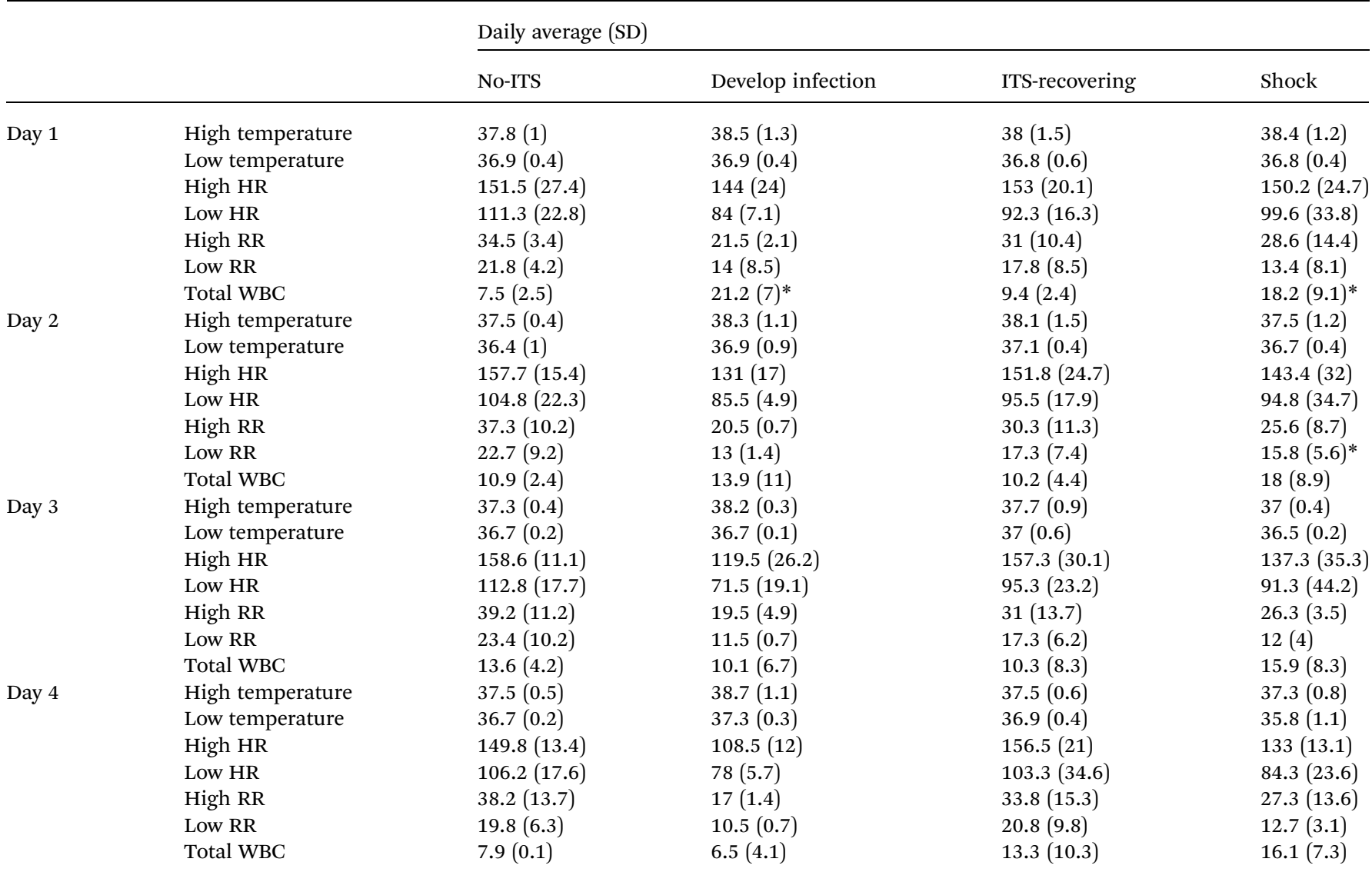

${ }^{a} \mathrm{HR}=$ heart rate, $\mathrm{RR}=$ respiratory rate, $\mathrm{WBC}=$ white blood cell count, and ITS $=$ infection, trauma or surgical. * indicates $p<0.05 v s$. No-ITS on the same day.

ventilated patients and may be a marker for changes in metabolism during the onset of infection and septic episodes.

We examined the relationship between the breath delta value and a diagnosis of SIRS and SIRS with shock. We found a lower, though not statistically significant, the BDV in subjects with SIRS compared to subjects with No-SIRS or SIRS with shock. The pediatric definition of SIRS is based on clinical parameters (principally body temperature and WBC count) that are not specific to infection. ${ }^{18}$ Spikes in temperature may be related to infection, but may also be related to trauma, burns or other diagnoses. ${ }^{18}$ As infections progress increasing body temperature and changing WBC counts are reliable markers, however, by the time changes in body temperature and WBC counts can be observed the infection has had systemic effects. ${ }^{21}$ The changes in macronutrient oxidation that occur as a result of changing physiology during sepsis and septic shock can cause dramatic changes in the BDV..$^{13,15-17,22,23}$ During severe sepsis peripheral tissues do not utilize oxygen well even with an abundant supply, thus increases in plasma lactic acid are a hallmark sign of sepsis..$^{15,23-26}$ The effect on whole body macronutrient metabolism is that lipid oxidation is limited and anaerobic glycolysis dominates. ${ }^{24}$ During normal metabolism lipid oxidation may contribute between 40 and $60 \%$ of the body's caloric need. The isotopic signature of lipids is much lighter than that of proteins or carbohydrates due to an enzymatic discrimination against ${ }^{13} \mathrm{C}$-structures by pyruvate dehydrogenase; ${ }^{11}$ thus reduced lipid oxidation causes a positive shift in the BDV. This effect has been observed in animal studies, ${ }^{13,16}$ and is evident in the SIRS with shock groups (Fig. 4, panel A). An individual case of sepsis transitioning into septic shock and death is presented in the ESI (Fig. S4) where the BDV can be seen transitioning from $-25.2 \%$ during sepsis to $-16.9 \%$ just prior to death due to septic shock. These data are consistent with impaired oxidative metabolism, particularly in hemodynamically unstable subjects who die within the first 24 hours of septic shock. ${ }^{25}$

Significantly lower breath delta values were observed in subjects with active sepsis without shock compared with those with No-ITS Fig. 4, panel B). This difference in breath delta value could be explained in part by fractionation of carbon during the catabolic inflammatory APR to infection (Fig. 1). ${ }^{9}$ Invading pathogens stimulate the innate immune response by activating tissue macrophages. Inflammatory cytokines such as tumor necrosis factor (TNF) and interleukin 1 (IL-1) are released by macrophages into the circulation. ${ }^{27,28}$ TNF and IL-1 induce catabolism and amino acid release from skeletal muscle and 
stimulate acute phase protein synthesis in the liver. ${ }^{29,30}$ Free amino acids are either used to build acute phase proteins or oxidized, through a series of reactions and expired as $\mathrm{CO}_{2}$. Due to the kinetic isotope effect, metabolites with heavier carbon $\left({ }^{13} \mathrm{C}\right.$, naturally abundant at $1 \%$ of carbon) move more slowly through enzymatic reactions. ${ }^{31}$ Since only one reaction is required to allocate an amino acid to protein synthesis (i.e. loading onto tRNA) there is an equal chance of this allocation for amino acids containing ${ }^{12} \mathrm{C}$ and ${ }^{13} \mathrm{C}$ structures. However, as the number of steps in the metabolic cascade increases, metabolites containing ${ }^{13} \mathrm{C}$ increasingly reside within the body while relatively more ${ }^{12} \mathrm{C}$ is expired in breath $\mathrm{CO}_{2} \cdot{ }^{31}$ Thus fractionation occurs during a catabolic response coupled with high rates of protein synthesis as seen in the APR to infection and to an extent trauma.

Analysis of the trends in the delta value may provide insight into the changing metabolism during infection and sepsis. In subjects with No-ITS the BDV remained stable in the -18 to $-19 \%$ range (Fig. 5). As previously postulated, the BDV in subjects that have undergone septic shock may have a complicated and variable response in the BDV, making interpretation of BDV in this population difficult. In our study, subjects who had undergone septic shock had a similar BDV to No-ITS subjects, making the BDV of limited value a marker for septic shock (Fig. 5). In subjects that did have an infection, trauma or surgery and were on a recovering clinical trajectory the BDV increased over time (Fig. 5). Finally in subjects who developed infections during the study, the BDV was initially similar to NoITS subjects, but trended down with the onset of infection. The BDV was significantly lower than the BDV of No-ITS subjects after the onset of infection (Fig. 5). Similarly the SIRS criteria were plotted in aggregate over time and evaluated. The SIRS criteria segregated patients who developed septic shock based on altered respiratory rates and WBC counts on the first or second day (Table 2). In comparison the BDV did not segregate septic shock subjects from No-ITS subjects (Fig. 5), but the BDV did segregate patients who developed infections during the study from those who did not based on the trend of the BDV over time (Fig. 5).

\section{Conclusion}

The BDV does not correlate well with the SIRS status. However, when patients are classified based on their inflammatory APR the breath delta value correlates with the severity of systemic inflammation. When monitored over time, changes in the BDV may correlate with changes in physiology related to fractionation during the APR to infection, trauma or due to altered macronutrient oxidation during episodes of septic shock.

\section{Acknowledgements}

We thank the Respiratory Therapy Department at the University of Wisconsin Hospital and Clinics for their participation in this project. We would like to thank Glen Leverson for consulting with us for statistical analysis of the data. Isomark, LLC, (Madison, WI) provided partial funding support for this study.

\section{References}

1 R. A. Balk, F. B. Cerra, R. P. Dellinger, A. M. Fein, W. A. Kanus, R. M. H. Schein and W. J. Sibbald, Crit. Care Med., 1992, 20, 864-874.

2 F. Proulx, M. Fayon, C. A. Farrell, J. Lacroix and M. Gauthier, Chest, 1996, 109, 1033-1037.

3 R. S. Watson, J. A. Carcillo, W. T. Linde-Zwirble, G. Clermont, J. Lidicker and D. C. Angus, Am. J. Respir. Crit. Care Med., 2003, 167, 695-701.

4 A. E. Jones, A. Focht, J. M. Horton and J. A. Kline, Chest, 2007, 132, 425-432.

5 B. Andreola, S. Bressan, S. Callegaro, A. Liverani, M. Plebani and L. Da Dalt, Pediatr. Infect. Dis. J., 2007, 26, 672-677.

6 J. N. Deis, C. B. Creech, C. M. Estrada and T. J. Abramo, Pediatric Emergency Medicine, 2010, 26, 51-60.

7 L. Simon, F. Gauvin, D. K. Amre, P. Saint-Louis and J. Lacroix, Clin. Infect. Dis., 2004, 39, 206-217.

8 M. Hatherill, S. M. Tibby, K. Sykes, C. Turner and I. A. Murdoch, Arch. Dis. Child., 1999, 81, 417-421.

9 D. E. Butz, M. E. Cook, H. R. Eghbalnia, F. Assadi-Porter and W. P. Porter, Rapid Commun. Mass Spectrom., 2009, 23, 37293735 .

10 K. A. Hatch, K. A. Sacksteder, I. W. Treichel, M. E. Cook and W. P. Porter, Biochem. Biophys. Res. Commun., 1995, 212, 719-726.

11 M. J. DeNiro and S. Epstein, Science, 1977, 197, 261-263.

12 W. P. Porter, M. E. Cook, F. Assadi-Porter and D. E. Bütz, Identification of disease characteristics using isotope ratios in breath. US Pat., US7465276, 2008.

13 D. E. Butz, S. L. Morello, J. Sand, J. P. Boriosi, G. N. Holland and M. E. Cook, FASEB J., 2013, 27, 868.

14 E. H. Wahl, B. Fidric, C. W. Rella, S. Koulikov, B. Kharmalov, S. Tan, A. A. Kachanov, B. A. Richman, E. R. Crosson, B. A. Paldus, S. Kalaskar and D. R. Bowling, Applications of cavity ring-down spectroscopy to high precision isotope ratio measurement of ${ }^{13} \mathrm{C} /{ }^{12} \mathrm{C}$ in carbon dioxide, Isot. Environ. Health Stud., 2006, 42, 21-35.

15 B. Goldstein, B. Giroir and A. Randolph, International pediatric sepsis consensus conference, Pediatr. Crit. Care Med., 2005, 6, 2-8.

16 D. E. Butz, W. P. Porter, F. Assadi-Porter and M. E. Cook, Early detection of the acute phase response in sepsis by identification of small molecule metabolites in breath and biofluids, FASEB J., 2010, 24, 752.4.

17 D. A. Schoeller, C. Brown, K. Nakamura, A. Nakagawa, R. S. Mazzeo, G. A. Brooks and T. F. Budinger, Biomed. Mass Spectrom., 1984, 11, 557-561.

18 B. Goldstein, B. Giroir and A. Randolph, Pediatr. Crit. Care Med., 2005, 6, 2-8.

19 R. C. Littell, J. Pendergast and R. Natarajan, Stat. Med., 2000, 19, 1793-1819. 
20 D. C. Angus, W. T. Linde-Zwirble, J. Lidicker, G. Clermont, J. Carcillo and M. R. Pinsky, Crit. Care Med., 2001, 29, 1303-1310.

21 R. P. Dellinger, M. M. Levy, J. M. Carlet, J. Bion, M. M. Parker, R. Jaeschke, K. Reinhart, D. C. Angus, C. Brun-Buisson, R. Beale, T. Calandra, J. F. Dhainaut, H. Gerlach, M. Harvey, J. J. Marini, J. Marshall, M. Ranieri, G. Ramsay, J. Sevransky, B. T. Thompson, S. Townsend, J. S. Vender, J. L. Zimmerman and J. L. Vincent, Crit. Care Med., 2008, 36, 296-327.

22 K. A. Hatch, B. Pinshow and J. R. Speakman, J. Comp. Physiol., B, 2002, 172, 263-268.

23 J. J. Spitzer, Circ. Shock Suppl., 1979, 1, 69-79.

24 J. P. Revelly, L. Tappy, A. Martinez, M. Bollmann, M. C. Cayeux, M. M. Berger and R. L. Chiolero, Crit. Care Med., 2005, 33, 2235-2240.
25 B. Levy, L. O. Sadoune, A. M. Gelot, P. E. Bollaert, P. Nabet and A. Larcan, Crit. Care Med., 2000, 28, 114-119.

26 E. Rivers, B. Nguyen, S. Havstad, J. Ressler, A. Muzzin, B. Knoblich, E. Peterson and M. Tomlanovich, N. Engl. J. Med., 2001, 345, 1368-1377.

27 I. De Freitas, M. Fernandez-Somoza, E. Essenfeld-Sekler and J. E. Cardier, Chest, 2004, 125, 2238-2246.

28 K. J. Tracey, B. Beutler, S. F. Lowry, J. Merryweather, S. Wolpe, I. W. Milsark, R. J. Hariri, T. J. Fahey, A. Zentella, J. D. Albert, G. T. Shires and A. Cerami, Science, 1986, 234, 470-474.

29 C. H. Lang, R. A. Frost and T. C. Vary, Am. J. Physiol.: Endocrinol. Metab., 2007, 293, E453-E459.

30 Y.-P. Li, Y. Chen, J. John, J. Moylan, B. Jin, D. L. Mann and M. B. Reid, FASEB J., 2005, 19, 362-370.

31 W. W. Cleland, J. Biol. Chem., 2003, 278, 51975-51984. 